\title{
A Generalised Hypergeometric Function
}

\author{
By Whltam Fabtan \\ (Received 1st November, 1949.)
}

\section{Introduction.}

The hypergeometric function ${ }^{1} F(a, b ; c ; z)$ is analytic in the domain $|\arg (-z)|<\pi$, and, when $|z|<1$, may be represented by the series

$$
\frac{\Gamma(c)}{\Gamma(a) \Gamma(b)} \sum_{n=0}^{\infty} \frac{\Gamma(a+n) \Gamma(b+n)}{\Gamma(c+n) \cdot n !} z^{n}
$$

When $|z|=1$ in the domain $|\arg (-z)|<\pi$, this series converges ${ }^{2}$ to $F(a, b ; c ; z)$ if $R(a+b-c)<0$ (integral values of $a, b$ and $c$ are excluded in the present paper).

This function belongs to a more general class of functions which may be represented, under certain conditions, by the series

$$
\frac{\Gamma(c) \Gamma(\lambda+1)}{\Gamma(a) \Gamma(b)} \sum_{n=-\infty}^{\infty} \frac{\Gamma(a+n) \Gamma(b+n)}{\Gamma(c+n) \Gamma(\lambda+n+1)} z^{\lambda+n} .
$$

For a discussion of this class of functions, fractional integrals will be employed.

\section{Fractional integrals.}

A $\lambda$-th integral of $F(a, b ; c ; z)$ along a simple curve $l$ from 0 to $z$ is defined $^{3}$ by

$$
D^{-\lambda}\left(l_{0}\right) F(a, b ; c ; z)=\frac{1}{\Gamma(\lambda+\gamma)}\left(\frac{d}{d z}\right)^{\gamma} \int_{0}^{z}(z-t)^{\lambda+\gamma-1} F(a, b ; c ; t) d t,
$$

where $\gamma$ is the least non-negative integer such that $R(\lambda)+\gamma>0$; the integration and differentiation being along $l$.

THEOREM 1. If l lies in $|z|<1$, then

$$
D^{-\lambda}\left(l_{0}\right) F(a, b ; c ; z)=\frac{\Gamma(c)}{\Gamma(a) \Gamma(b)} \sum_{n=0}^{\infty} \frac{\Gamma(a+n) \Gamma(b+n)}{\Gamma(c+n) \Gamma(\lambda+n+1)} z^{\lambda+n} .
$$

1 Whittaker and Watson, Modern Analysis (1927), Ch. XIV.

2 Ibid., pp. 25 and 57.

${ }^{3}$ Fabian, Quart. J. of Math., 7 (1936), 252. Cf. the Riemann-Liouville integral. 
This equality continues to hold when $|z|=1$ in the domain $|\arg (-z)|<\pi$, provided that $R(a+b-c-\lambda)<0$.

Proof. The first part of the theorem follows immediately by applying the operator $D^{-\lambda}$ to each term of the series

$$
F(a, b ; c ; z)=\frac{\Gamma(c)}{\Gamma(a) \Gamma(b)} \sum_{n=0}^{\infty} \frac{\Gamma(a+n) \Gamma(b+n)}{\Gamma(c+n) \cdot n !} z^{n}
$$

To prove the second part of the theorem, we note first that each branch of $D^{-\lambda}\left(l_{0}\right) F(a, b ; c ; z)$ is analytic in and on the circle $|z|=1$ in the domain $|\arg (-z)|<\pi, z \neq 0$, since $F(a, b ; c ; z)$ is analytic in this region'. The required conclusion will then follow if we prove that the series stated in the theorem converges when $|z|=1,|\arg (-z)|<\pi$, and $R(a+b-c-\lambda)<0$.

To prove this, denote the $n$-th term of this series by $u_{n}$. Then we have, when $|z|=1$,

$$
\begin{aligned}
\left|\frac{u_{n+1}}{u_{n}}\right| & =\left|\frac{(a+n-1)(b+n-1)}{(c+n-1)(\lambda+n)}\right| \\
& =\left|1+\frac{a+b-c-\lambda-1}{n}+O\left(\frac{1}{n^{2}}\right)\right| \\
& =\left|1+\frac{R(a+b-c-\lambda)-1}{n}+O\left(\frac{1}{n^{2}}\right)\right|
\end{aligned}
$$

Hence, by a known theorem ${ }^{2}$, this series converges absolutely when $|z|=1$, if $R(a+b-c-\lambda)<0$.

This completes the proof.

\section{The more general class of functions.}

THeOREM 2. For non-integral values of $a, b, c$ and $\lambda$, there exists $a$ function $S(a, b ; c, \lambda ; z)$ which consists of branches analytic in the finite part of the domain $|\arg (-z)|<\pi, z \neq 0$; and which, when $|z|=1$ in this domain and $R(a+b-c-\lambda)<0$, may be represented by the series

$$
\frac{\Gamma(c) \Gamma(\lambda+1)}{\Gamma(a) \Gamma(b)} \sum_{n=-\infty}^{\infty} \frac{\Gamma(a+n) \Gamma(b+n)}{\Gamma(c+n) \Gamma(\lambda+n+1)} z^{\lambda+n} .
$$

1 Fabian, Math. Gazette, 20 (1936), 249.

2 Whittaker and Watson, op. cit., p. 23. 
Proof. This series may be written

$$
\begin{aligned}
& \frac{\Gamma(c) \Gamma(\lambda+1)}{\Gamma(a) \Gamma(b)} \sum_{n=0}^{\infty} \frac{\Gamma(a+n) \Gamma(b+n)}{\Gamma(c+n) \Gamma(\lambda+n+1)} z^{\lambda+n} \\
& \quad+\sum_{n=1}^{\infty} \frac{(c-1)(c-2) \ldots(c-n) \lambda(\lambda-1) \ldots\left(\lambda-\frac{n+1)}{(a-1)(a-2) \ldots(a-n)(b-1)(b-2)}\right.}{\cdot(b-n)} z^{\lambda-n}
\end{aligned}
$$

that is

$$
\begin{gathered}
\frac{\Gamma(c) \Gamma(\lambda+1)}{\Gamma(a) \Gamma(b)} \sum_{n=0}^{\infty} \frac{\Gamma(a+n) \Gamma(b+n)}{\Gamma(c+n) \Gamma(\lambda+n+1)} z^{\lambda+n} \\
+z^{\lambda-a} \sum_{n=1}^{\infty} \frac{(-c+1)(-c+2) \ldots(-c+n)(-\lambda)(-\lambda+1) \ldots(-\lambda+n-1)}{(-a+1)(-a+2) \ldots(-a+n)(-b+1)(-b+2) \ldots(-b+n)}\left(\frac{1}{z}\right)^{n-a} .
\end{gathered}
$$

By Theorem 1, this represents the function

$$
\begin{aligned}
\Gamma(\lambda+1) D^{-\lambda}\left(l_{0}\right) F(a, b ; c ; z) & \\
& +\Gamma(1-a) \cdot z^{\lambda-a} D^{a}\left(L_{0}\right) F(1-c,-\lambda ; 1-b ; w)-z^{\lambda}
\end{aligned}
$$

when $|z|=1,|\arg (-z)|<\pi$, and $R(a+b-c-\lambda)<0 ; w$ being $1 / z$, and $L$ the path of integration in the $w$-plane.

If we denote this function by $S(a, b ; c, \lambda ; z)$, each branch of $S(a, b ; c, \lambda ; z)$ is analytic in the finite part of the domain $|\arg (-z)|<\pi$, $z \neq 0$, by a previous theorem ${ }^{1}$.

Hence the conclusion.

1 Fabian, Math. Gazette, 20 (1936), 249.

14 Grosvenor Avenue,

Canonbury, London, N.5. 